\title{
Optimizing Nanoparticle Designs for Ideal Absorption of Light
}

\author{
Victor Grigoriev, Nicolas Bonod, Jérôme Wenger, and Brian Stout* \\ Aix-Marseille Université, CNRS, Centrale Marseille, Institut Fresnel, UMR 7249, 13397 Marseille, France
}

\begin{abstract}
Resonant interaction of light with nanoparticles is essential for a broad range of nanophotonics and plasmonics applications, including optical antennas, photovoltaics, thermoplasmonics, and sensing. Given this broad interest, analytical formulas are highly desirable to provide design guidelines for reaching the conditions of ideal absorption. Here we derive analytical expressions to accurately describe the electric and magnetic modes leading to ideal absorption. Our

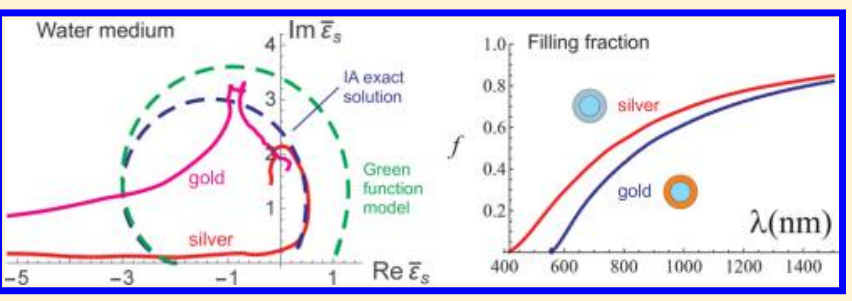
model significantly improves on accuracy as compared to classical models using Green's functions or a Mie coefficient expansion. We demonstrate its applicability over a broad parameter space of frequencies and particle diameters up to several wavelengths. We reveal that ideal absorption is attainable in homogeneous spherical nanoparticles made of gold or silver at specific sizes and illumination frequencies. To reach ideal absorption at virtually any frequency in the visible and near-infrared range, we provide explicit guidelines to design core-shell nanoparticle absorbers. This work should prove useful for providing experimental designs that optimize absorption and for a better understanding of the physics of ideal absorption.
\end{abstract}

KEYWORDS: ideal absorption, coherent perfect absorption, plasmonics, scattering theory, Mie theory, optical magnetic modes, resonant photonics

$\mathrm{M}$ etallic nanoparticles and, more recently, dielectric nanoparticles are receiving tremendous attention due to their ability to concentrate light energy into volumes at the nanometer scale. ${ }^{1,2}$ Light-matter interaction around nanoparticles is at the core of a wide range applications including optical antennas, ${ }^{3}$ light harvesting, ${ }^{4}$ thermoplasmonics, ${ }^{5}$ and local surface plasmon resonance sensing. ${ }^{6}$ In this context, optimizing the design of nanoparticles to maximize absorption of light is of crucial importance. ${ }^{7,8}$ This will lead to enhanced light-matter interaction, photoluminescence, or heat generation.

While brute force numerical simulations can certainly provide reliable design parameters, they come at the price of extensive parameter searches and a lack of physical insight. Analytical expressions for simple and faultless nanoparticle geometries are therefore interesting, as they are quite straightforward for other researchers to use and provide insights into light absorption problems. However, as we will show below, classical point-like dipole models are inaccurate for nanoparticles of sizes $\gtrsim 30 \mathrm{~nm}$, and a more realistic model needed to be developed.

In this manuscript, we develop a conceptual framework to achieve ideal absorption of light by metallic and dielectric nanoparticles over a broad parameter space of frequencies and particle sizes. We define Ideal Absorption (IA) as the conditions leading to a mode of the total field being completely absorbed by the nanoparticle. Ideal absorption is also sometimes referred to as "coherent perfect absorption". 9-11 However, it should be pointed out that absorption of the incident field is only perfect for an ideal $4 \pi$ illumination specifically tailored to the IA mode. ${ }^{12}$
As our main result, eq 16 provides explicitly analytical formulas describing the conditions to reach IA for homogeneous spherical nanoparticles. For such particles, only a restricted number of attainable parameters (radius, permittivity, frequency) reach ideal absorption. We proceed to show that core-shell nanoparticles feature the ability to satisfy the ideal light absorption condition over the full visible spectrum by tuning the filling factor and the radius of the nanoparticle. We provide explicit guidelines (eq 18) to optimize the design so as to reach ideal absorption for silver and gold core-shell nanoparticles.

The manuscript is organized as follows: in Section 1, we introduce ideal absorption and demonstrate that it corresponds to a fundamental upper bound in an absorption cross section channel ( $\sigma_{\mathrm{a}}=3 \lambda^{2} / 8 \pi$ for dipole resonators). We then discuss in Section 2 two classical "point-like" models providing analytical formulas for predicting fundamental electric dipole IA (eq 12). Since these models are limited to treatments of fundamental electric modes and make rather inaccurate predictions for all but the smallest particle sizes, we derive significantly improved analytic formulas in eq 16 predicting the required electric and magnetic fundamental dipole IA solutions in homogeneous scatterers. Section 3 shows numerical results using a Weierstrass factorization method ${ }^{13,14}$ that determines fundamental and higher order IA modes to arbitrary accuracy. After some discussions and calculations concerning multimode absorption and IA bandwidth, Section 4 illustrates some predictions for IA in homogeneous spheres with gold and silver at specific frequencies and sizes. Finally, the discussion is

Received: October 4, 2014 
extended to core-shell nanoparticles (Section 5). Combining eq 18 with eq $16 \mathrm{a}$, we provide a simple procedure to design optimized core-shells achieving IA at essentially any frequency in the visible and near visible range.

\section{SCATTERING THEORY FOR IDEAL ABSORPTION}

Scattering in three-dimensional electromagnetic problems is conveniently expressed in terms of incoming and outgoing spherical Vector Partial Waves (VPWs). ${ }^{15}$ The VPWs are solutions of homogeneous media Maxwell equations of either electric source type $\left(e: \boldsymbol{N}_{n, m}^{( \pm)}(k \mathbf{r})\right)$ or magnetic source type $(h$ : $\left.\boldsymbol{M}_{n, m}^{( \pm)}(k \mathbf{r})\right)$, with the $+(-)$ superscripts indicating that the functions satisfy outgoing (incoming) boundary conditions, respectively. The subscript, $n$, denotes the total angular momentum number, and $m=-n, \ldots, n$, the angular momentum projection number. ${ }^{15}$ The spatial dependence of the VPWs is scaled by the in-medium wavenumber, $k=\left(\varepsilon_{\mathrm{b}} \mu_{\mathrm{b}}\right)^{1 / 2} \omega / c=$ $N_{\mathrm{b}} \omega / c=2 \pi / \lambda$, where $N_{\mathrm{b}}$ is the refraction index of the homogeneous background material, and $\lambda$, the in-medium wavelength.

Theoretical treatments of electromagnetic scattering from a homogeneous spherically symmetric particle go under the names of Lorenz-Mie-Debye theory, but the physical content is best viewed in the formalism of $\mathrm{S}$ or $\mathrm{T}$ matrices, originally developed for quantum mechanical scattering theory. ${ }^{19-19,22}$ The S-matrix seems best adapted to the discussion of IA and is privileged from here on.

The total field in a homogeneous region surrounding a particle can always be developed on the basis set of the incoming and outgoing VPWs, with $a_{n, m}^{(e, \pm)}$ and $a_{n, m}^{(h, \pm)}$, respectively, denoting the electric and magnetic mode VPWs field coefficients. Since the S-matrix of a spherically symmetric system is automatically diagonal in the VPW basis, its elements express the linear relationship between the outgoing and incoming field coefficients of the total field for both electric and magnetic source fields respectively, that is, $S_{n}^{(e)}=a_{n, m}^{(e,+)} / a_{n, m}^{(e,-)}$, and $S_{n}^{(h)}=a_{n, m}^{(h,+)} / a_{n, m}^{(h,-)}$.

Algebraic manipulations involving the T-matrix, ${ }^{16,15}$ and the definition of the S-matrix, ${ }^{15,18} \overline{\bar{S}} \equiv \overline{\bar{I}}+2 \overline{\bar{T}}$, provide convenient expressions for the $S$-matrix coefficients:

$$
\begin{aligned}
& S_{n}^{(e)}=-\frac{h_{n}^{(-)}(k R)}{h_{n}^{(+)}(k R)} \cdot \frac{\bar{\varepsilon}_{s} \varphi_{n}^{(-)}(k R)-\varphi_{n}\left(k_{\mathrm{s}} R\right)}{\bar{\varepsilon}_{\mathrm{s}} \varphi_{n}^{(+)}(k R)-\varphi_{n}\left(k_{\mathrm{s}} R\right)} \\
& S_{n}^{(h)}=-\frac{h_{n}^{(-)}(k R)}{h_{n}^{(+)}(k R)} \cdot \frac{\bar{\mu}_{s} \varphi_{n}^{(-)}(k R)-\varphi_{n}\left(k_{s} R\right)}{\bar{\mu}_{\mathrm{s}} \varphi_{n}^{(+)}(k R)-\varphi_{n}\left(k_{\mathrm{s}} R\right)}
\end{aligned}
$$

where, $\bar{\varepsilon}_{\mathrm{s}} \equiv \varepsilon_{\mathrm{s}} / \varepsilon_{\mathrm{b}}$, and $\bar{\mu}_{\mathrm{s}} \equiv \mu_{\mathrm{s}} / \mu_{\mathrm{b}}$ are, respectively, the permittivity and permeability contrasts between the sphere and the host medium, and $k_{\mathrm{s}}$ is the wavenumber inside the sphere. The $h_{n}^{(+)}(x)$ and $h_{n}^{(-)}(x)$ functions, respectively, denote the outgoing and incoming spherical Hankel functions. The expressions in eq 1 also employ the functions:

$$
\varphi_{n}^{( \pm)}(x) \equiv \frac{\left[x h_{n}^{( \pm)}(x)\right]^{\prime}}{h_{n}^{( \pm)}(x)}, \quad \varphi_{n}(x) \equiv \frac{\left[x j_{n}(x)\right]^{\prime}}{j_{n}(x)}
$$

where $j_{n}(x)$ are the $n^{\text {th }}$ order spherical Bessel functions.

Flux conservation imposes an upper bound on the amplitude of the $S$ matrix elements, $\left|S_{n}^{(e, h)}\right| \leq 1$, where the upper limit, $\left|S_{n}^{(e, h)}\right|=1$ is satisfied by any lossless scatterer since this condition results directly in $\left|a_{n, m}^{(+)}\right|=\left|a_{n, m}^{(-)}\right|{ }^{18}$ The S-matrix of a lossless scatterer is characterized by zeros (absorbing modes) in the upper-half complex frequency plane, and poles (emitting modes) in the lower-half plane frequency plane. ${ }^{13,14}$ Adding absorption to the particle causes the absorbing modes to descend toward the lower complex plane with IA occurring at those values of $\bar{\varepsilon}_{\mathrm{s}}$ for which a zero of the S-matrix lies on the real frequency axis, that is, there exists an $n$ such that

$$
\begin{aligned}
& S_{n}^{(e)}=0 \Leftrightarrow \frac{\varphi_{n}\left(k_{\mathrm{s}} R\right)}{\bar{\varepsilon}_{\mathrm{s}}}=\varphi_{n}^{(-)}(k R) \text { or } \\
& S_{n}^{(h)}=0 \Leftrightarrow \frac{\varphi_{n}\left(k_{\mathrm{s}} R\right)}{\bar{\mu}_{\mathrm{s}}}=\varphi_{n}^{(-)}(k R)
\end{aligned}
$$

when $\mathcal{I}\{\omega\}=0$ (these conditions being found by inspection of eqs $1 \mathrm{a}$ and $1 \mathrm{~b}$.

Scattering and absorption cross sections of a spherically symmetric scatterer are the sum of the contributions from all multipole orders, $n$ :

$$
\sigma_{\mathrm{s}}=\sum_{n=1}^{\infty}\left(\sigma_{n, \mathrm{~s}}^{(e)}+\sigma_{n, \mathrm{~s}}^{(h)}\right), \quad \sigma_{\mathrm{a}}=\sum_{n=1}^{\infty}\left(\sigma_{n, \mathrm{a}}^{(e)}+\sigma_{n, \mathrm{a}}^{(h)}\right)
$$

The multipole contributions to the absorption, scattering, and extinction cross sections ${ }^{15,18,19}$ can be, respectively, expressed in terms of the S-matrix as

$$
\begin{aligned}
& \sigma_{n, \mathrm{a}}^{(e, h)}=\frac{\pi}{2 k^{2}}(2 n+1)\left(1-\left|S_{n}^{(e, h)}\right|^{2}\right) \\
& \sigma_{n, \mathrm{~s}}^{(e, h)}=\frac{\pi}{2 k^{2}}(2 n+1)\left|S_{n}^{(e, h)}-1\right|^{2} \\
& \sigma_{n, \mathrm{e}}^{(e, h)}=\frac{\pi}{k^{2}}(2 n+1) \mathcal{R}\left\{1-S_{n}^{(e, h)}\right\}
\end{aligned}
$$

where the $2 n+1$ factors arise from the azimuthal mode degeneracy of the orbital modes.

One finds that an IA mode contributes equally to the absorption and scattering cross sections by inserting the IA criteria, $S_{n}=0$ into eqs $5 \mathrm{a}$ and $5 \mathrm{~b}$ (in either the $(e)$ or $(h)$ channels), with values of ${ }^{20,21}$

$$
\sigma_{n, \mathrm{~s}}=\sigma_{n, \mathrm{a}}=\frac{2 n+1}{8 \pi} \lambda^{2}
$$

which is an upper bound for the absorption cross section of a mode, but a factor four times less than the $S_{n}=-1$, unitary limit $^{17}$ of the scattering or extinction cross sections, obtainable from eqs $5 b$ and $5 c$. The equality of scattering and absorption cross sections in an IA channel follows from the fact that IA requires the field scattered by the particle to have perfectly destructive interference with the outgoing wave components of the local excitation field (leaving the $n^{\text {th }}$ order modes of the total field to be purely incoming). We also remark that many properties of IA in particles, like eq 6, find analogues in the ideal absorption of 1D systems, where for example the maximum absorption coefficient, $A$, of a plane wave illuminating a single side of a symmetric thin film occurs when $A=R+T=0.5$. $^{23,24}$

Since the $\varphi_{n}^{(-)}(k R)$ is a complex function of a real variable, and $\varphi_{n}\left(k_{\mathrm{s}} R\right)$ is real for real value $k_{\mathrm{s}}$, the IA solutions of eqs $3 \mathrm{a}$ or $3 \mathrm{~b}$ can occur only for complex values of $\bar{\varepsilon}_{\mathrm{s}}$. Furthermore, eqs 3 are transcendental equations, and each has an infinite number of solutions. For the sake of clarity, further discussion in this 


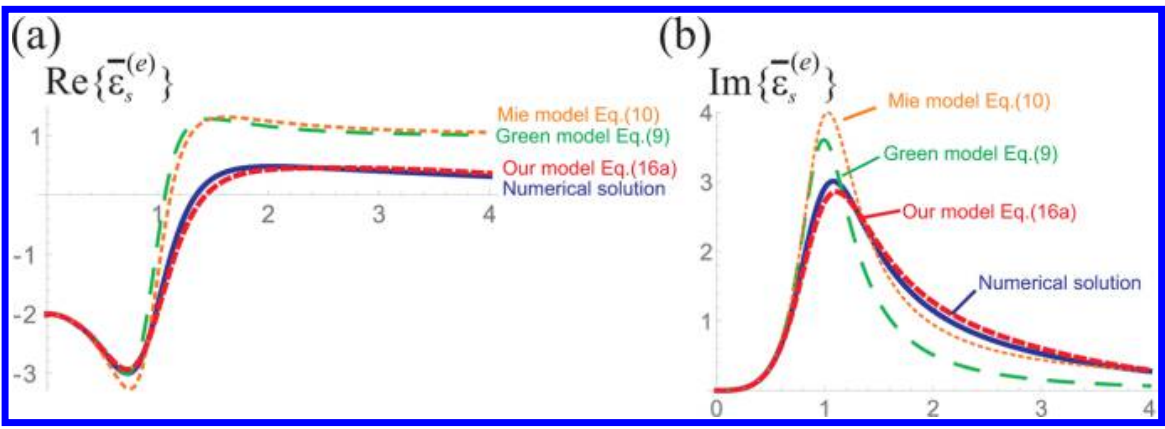

Figure 1. Numerical results of the real and imaginary parts of $\bar{\varepsilon}_{\mathrm{s}}$, yielding the lowest IA solution are plotted as a function of $k R$ (blue solid curves). The predictions from the Green function point-like model eq 9 are plotted in green (long dashed lines), and the Mie theory development model eq 10 are plotted in orange (dotted lines). Our analytical model eq 16a (red short dashed curves) provides an improved description of the conditions to reach ideal absorption over a broad $k R$ range.

work will be limited to the $n=1$ (i.e., dipole) mode solutions of eq 3 .

The real and imaginary parts of the solutions of eq 3a with the lowest values of $\mathcal{R}\left\{\bar{\varepsilon}_{\mathrm{s}}\right\}$ are plotted as the blue (solid) line in Figure 1 for particle diameters ranging from zero up to a little more than a wavelength. These results were computed by taking advantage of the newly developed Weierstrass factorization for resonant photonic structures. ${ }^{13,14}$ One readily remarks that this solution tends to the well-known quasi-static dipolar plasmon resonance at $\bar{\varepsilon}_{\mathrm{s}}=-2$ when $k R \rightarrow 0$. When only this IA mode is of interest, one can try to describe IA with a "point-like" model aimed at providing approximate descriptions of the lowest $\mathcal{R}\left\{\bar{\varepsilon}_{\mathrm{s}}\right\}$ electric dipole resonance. ${ }^{25,26}$ This is the aim of the next section.

\section{POINT-LIKE MODELS AND ANALYTIC EXTENSIONS} Unitarity considerations ${ }^{15,17,18}$ dictate that the polarizability of a small isotropic scatterer takes the form: ${ }^{27-29}$

$$
\alpha_{e}^{-1}=\alpha_{\mathrm{qs}}^{-1}-i \frac{k^{3}}{6 \pi}
$$

where the quasi-static polarizability, $\alpha_{\mathrm{qs}}$, is real in the absence of absorption, while the $-i k^{3} / 6 \pi$ term accounts for radiative losses. Unitarity, however, does not dictate the finite frequency corrections to $\alpha_{\mathrm{qs}}$, and one only knows that $\alpha_{\mathrm{qs}}$ must tend toward its quasi-static value in the small $k R$ limit:

$$
\lim _{k R \rightarrow 0} \alpha_{\mathrm{qs}}=4 \pi R^{3} \frac{\bar{\varepsilon}_{\mathrm{s}}-1}{\bar{\varepsilon}_{\mathrm{s}}+2} \equiv \alpha_{0}
$$

where $\alpha_{0}$ is the "static" polarizability of a sphere. Some authors simply impose $\alpha_{\mathrm{qs}}=\alpha_{0}$, while others, using first-principles Green's function theory and "regularizations" of the point-like integrals, commonly derive a $k^{2}$ order correction to $\alpha_{\mathrm{qs}}^{-1}$ of the form: ${ }^{26,30,31}$

$$
\left[\alpha_{\mathrm{qs}}^{(G . t .)}\right]^{-1} \simeq \alpha_{0}^{-1}-\frac{k^{2}}{6 \pi R}
$$

An alternative approach is to expand the exact Mie formula to lowest order in $k^{2}$, as proposed earlier by $\mathrm{Moroz}^{27}$ (see also Stratton $^{32}$ ), which leads to

$$
\left[\alpha_{\mathrm{qs}}^{(M . t .)}\right]^{-1} \simeq \alpha_{0}^{-1}-\frac{3}{5} \frac{\bar{\varepsilon}_{\mathrm{s}}-2}{\bar{\varepsilon}_{\mathrm{s}}-1} \frac{k^{2}}{4 \pi R}
$$

It is a bit surprising that the factors multiplying the $k^{2}$ corrections of eqs 9 and 10 do not agree. We attribute this to the lack of rigor in the regularizations of the Green function derivation. It seems nevertheless desirable to include $k^{2}$ corrections to the polarizability since such corrections predict the physically observed "red-shift" of localized plasmon resonances with increasing particle size.

Neither eq 9 nor eq 10 is strictly necessary for isotropic scatterers, since in this case the polarizability is directly related to the electric dipole S-matrix element, $S_{1}^{(e)}$, via the relation ${ }^{33}$

$$
\alpha_{e}=\frac{3 \pi i}{k^{3}}\left(1-S_{1}^{(e)}\right)
$$

thus, predicting an IA polarizability of $\alpha_{\mathrm{IA}}=3 \pi i / k^{3}$. The advantage of adopting a point-like model is that if one replaces the left-hand side of eq 7 with $\alpha_{\text {IA }}$, so that using either eq 9 or eq 10 , one can algebraically solve for the value of $\bar{\varepsilon}_{\mathrm{IA}}^{(e)}$ as a function of the size parameter, $\rho \equiv k R$ :

$$
\bar{\varepsilon}_{\mathrm{IA}}^{(e)}(\rho)=\left\{\begin{array}{cc}
-2 \frac{1+(1 / 3) \rho^{2}(1-i \rho)}{1-(2 / 3) \rho^{2}(1-i \rho)} & \text { green function: eq } 9 \\
-2 \frac{1+(1 / 15) \rho^{2}(9-5 i \rho)}{1-(1 / 15) \rho^{2}(9+10 i \rho)} & \text { Mie development: eq } 10
\end{array}\right.
$$

The real and imaginary parts of these functions are plotted in Figure 1a,b. Although both point-like predictions for $\bar{\varepsilon}_{\mathrm{IA}}^{(e)}(k R)$ tend toward the exact result (blue curve) in the $k R \rightarrow 0$ limit, they differ significantly from the exact result and each other for larger $k R$.

In view of the inaccuracies of these traditional point-like models, we derive below more reliable predictions of the IA conditions by analyzing the pole structure of the special functions. One first remarks that the $\varphi_{n}^{( \pm)}$functions appearing in eq 1 have finite meromorphic expressions, which for the dipole example take the form

$$
h_{1}^{( \pm)}(z)=-e^{ \pm i z} \frac{z \pm i}{z^{2}} ; \varphi_{1}^{( \pm)}(z)= \pm i z-\frac{1}{1 \mp i z}
$$

The $\varphi_{n}$ functions, on the other hand, have an infinite number of poles located along the real axis and can be expressed in meromorphic form as

$$
\varphi_{n}(z)=n+1+\sum_{\alpha=1}^{\infty}\left(\frac{2 z^{2}}{z^{2}-a_{n, \alpha}^{2}}\right)
$$

where the constants, $a_{n, \alpha}$, are the zeros of the spherical Bessel function, $j_{n}(x)$, and are tabulated. ${ }^{34}$ 
One can obtain analytic dipole approximations to eq $1 \mathrm{~b}$ by replacing $\varphi_{1}$ of eq 14 with an approximate meromorphic function having the same two lowest poles, $a$ and zeros, $b$ :

$$
\varphi_{1}(z) \approx 2 \frac{1-(z / b)^{2}}{1-(z / a)^{2}}
$$

where $a=1.4303 \pi$ and $b=0.87335 \pi$. Adopting this substitution transforms $S_{1}^{(e, h)}=0$ into quadratic equations in terms of $\bar{\varepsilon}_{\mathrm{s}}^{(e, h)}$ whose relevant solutions are

$$
\begin{aligned}
\bar{\varepsilon}_{\mathrm{IA}}^{(e)}(\rho)= & \frac{1}{2} \frac{a^{2}}{\rho^{2}}\left[1+\frac{\rho^{2}}{b^{2}} \frac{2(i \rho+1)}{\left(\rho^{2}-i \rho-1\right)}\right. \\
& -\sqrt{\left.\left(1+\frac{\rho^{2}}{b^{2}} \frac{2(i \rho+1)}{\left(\rho^{2}-i \rho-1\right)}\right)^{2}-\frac{\rho^{2}}{a^{2}} \frac{8(i \rho+1)}{\left(\rho^{2}-i \rho-1\right)}\right]} \\
\bar{\varepsilon}_{\mathrm{IA}}^{(h)}(\rho) & =\frac{3+3 i \rho-\rho^{2}}{\rho^{2}\left\{\left[(2+2 i \rho) / b^{2}\right]+\left[\left(1+i \rho-\rho^{2}\right) / a^{2}\right]\right\}}
\end{aligned}
$$

This set of analytical formulas forms our first main result. It allows the accurate design of ideal absorbers in both electric and magnetic modes, and can be immediately used by other researchers to optimize absorption in nanoparticles. The electric dipole solution of eq 16a is plotted in red in Figure 1. It remarkably converges toward the numerical exact solution (blue curve) over a broad $k R$ parameter range. The magnetic dipole solution of eq $16 \mathrm{~b}$ is plotted in Figure $1 \mathrm{c}, \mathrm{d}$, where it is visually indistinguishable from the numerically exact solution. Our new analytical formulas are thus valid up to particles sizes of several hundreds of nanometers, which constitutes an improvement of more than an order of magnitude in nanoparticle size with respect to classical "point-like" models.

\section{EXACT SOLUTIONS}

Although the analytic expressions of eq 16 provide accurate approximations for the lowest IA modes, one may nevertheless desire higher precision or the predictions of additional IA solutions, either of which will require numerical solutions of eq $3 \mathrm{a}$ or eq $3 \mathrm{~b}$. Finding these solutions is quite difficult when using commonly employed techniques involving Cauchy integrals in the complex plane or conjugate gradient methods, but they can be readily solved using techniques based on Weierstrass factorization, ${ }^{13,14}$ which exploit the meromorphic expansion of eq 14.

The real and imaginary parts of the numerically obtained dipole solutions of eqs $3 \mathrm{a}$ and $3 \mathrm{~b}$ for the $\bar{\varepsilon}_{\mathrm{s}}$ are plotted in 1 as a function of $k R$ for particles ranging in size from zero up to slightly larger than a wavelength $(k R=\pi$ corresponds to particle diameter $=\lambda$ ).

Even though the IA conditions can be found from the numerical solutions presented in Figure 2, absorber design will generally require taking into account the other properties of the absorber, like IA bandwidth and absorption in other modes. We illustrate this point with some plots of the dimensionless absorption cross section, $8 \pi \sigma_{\mathrm{abs}} /\left(3 \lambda^{2}\right)$, for particles designed to satisfy electric dipole IA at a few different subwavelength size parameters, notably $k R=0.3$ and $k R=1$ in Figure 3a,b. The total absorption cross sections are plotted in blue, the electric dipole absorption cross sections in red, and as a further piece of information, the magnetic dipole absorption cross section is plotted in green.

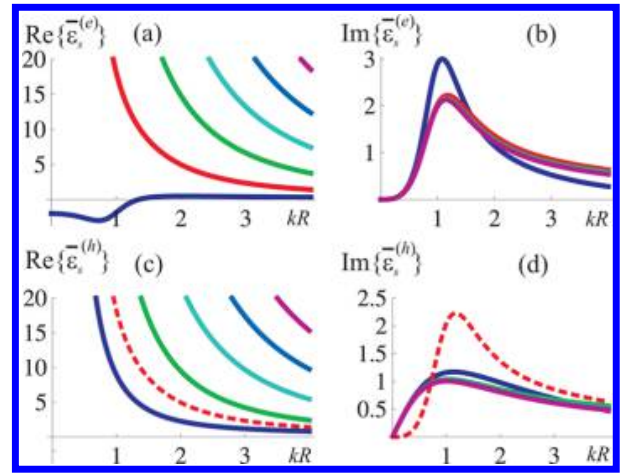

Figure 2. Conditions to reach electric dipole Ideal Absorption as a function of $k R$ for the real (a) and imaginary (b) part of the complex permittivity $\bar{\varepsilon}_{s}$. Magnetic dipole IA values for $\bar{\varepsilon}_{s}$ are also plotted in (c,d). The lowest electric dipole solution with $\mathcal{R}\left\{\bar{\varepsilon}_{\mathrm{s}}\right\}>1$ is plotted as a dashed curve in (c) and (d) for comparison.

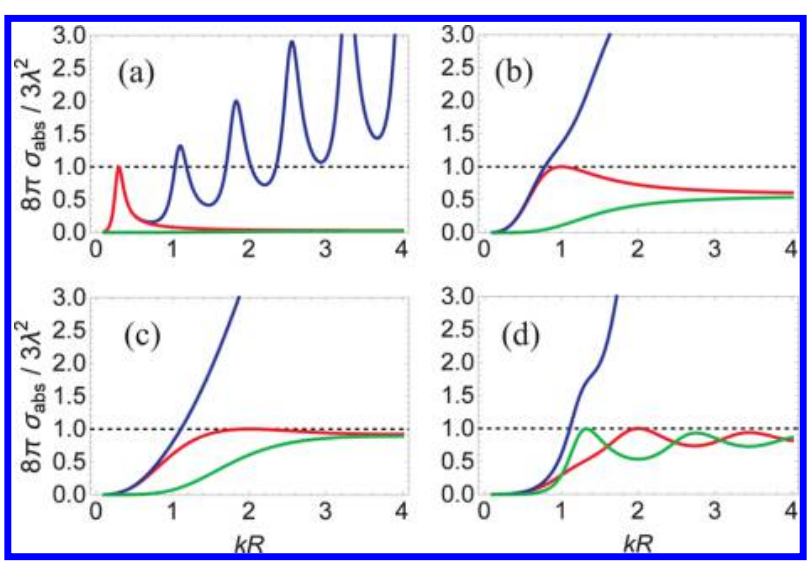

Figure 3. Dimensionless absorption cross sections, $8 \pi \sigma_{\mathrm{abs}} /\left(3 \lambda^{2}\right)$, plotted as a function of $k R$ for IA designed to occur at: (a) $k R=0.3, \bar{\varepsilon}_{\mathrm{s}}$ $=-2.21+i 0.0611$; (b) $k R=1, \bar{\varepsilon}_{\mathrm{s}}=-1.87+i 2.91$; (c) $k R=2, \bar{\varepsilon}_{\mathrm{s}}=$ $0.4809+i 1.148$; and (d) $k R=2, \bar{\varepsilon}_{\mathrm{s}}=4.968+i 1.361$. Total absorption cross sections are given by blue curves, electric dipole absorption by red curves, and magnetic dipole absorption by green curves.

One remarks from Figure 2 that IA can be satisfied for relatively modest values of $\mathcal{R}\left\{\bar{\varepsilon}_{\mathrm{s}}\right\}>1$ for size parameters roughly larger than unity (i.e., $D \gtrsim \lambda / 3$ ). For IA designed to occur at $k R=2$, absorption cross sections are plotted for both the $\mathcal{R}\left\{\bar{\varepsilon}_{\mathrm{s}}\right\}<1$ solution value of $0.4809+i 1.148$ in Figure $3 \mathrm{c}$ and the lowest $\mathcal{R}\left\{\bar{\varepsilon}_{\mathrm{s}}\right\}>1$ solution of $\bar{\varepsilon}_{\mathrm{s}}=4.968+i 1.361$ in Figure 3d.

For scatterers that are quite small with respect to the IA wavelength, like $k R=0.3$ in Figure $3 a$, one clearly observes peaks in the total absorption cross section associated with higher electric multipole modes, although only the electric dipole mode (red curve), satisfies the IA condition per its design. We remark that the magnetic dipole contributions (green curve) are insignificant at such sizes. For somewhat larger sub- $\lambda$ particles, like the $k R=1$ electric dipole IA solution in Figure $3 b$, one remarks that the electric dipole IA is accompanied by non-negligible absorption in higher order modes including the magnetic dipole contribution (in green). This behavior is accentuated for $k R=2$ with magnetic dipole contributions coming close to the IA condition in both the $\mathcal{R}\left\{\bar{\varepsilon}_{\mathrm{s}}\right\}<1$ and $\mathcal{R}\left\{\bar{\varepsilon}_{\mathrm{s}}\right\}>1$ designs. We remark, however, that even when IA occurs for both electric and magnetic dipole modes in the same particle, they cannot both be satisfied at the 
same frequency (for a homogeneous particle at least). We also underline the fact that for $\mathcal{R}\left\{\bar{\varepsilon}_{s}\right\}>1$ absorbers, it can be easier to obtain magnetic dipole IA solutions than those of electric dipoles. The ability for small particles to produce strong magnetic dipole absorption at optical frequencies has also been remarked recently by other authors. ${ }^{35}$

\section{IDEAL ABSORPTION WITH REALISTIC MATERIALS}

Materials exhibiting plasmonic resonances like silver and gold are good candidates to achieve ideal absorption at small nanoparticle sizes since they provide $\mathcal{R}\left\{\bar{\varepsilon}_{\mathrm{s}}\right\}<1$ and modest absorption over the visible and near visible frequencies. Even for these materials, however, ideal absorption will only occur at certain frequencies and sizes, and generally requires exploiting at least one tunable parameter like the refractive index $N_{\mathrm{b}}$ of the background medium.

We plot in Figure 4a, the path traced out by $\bar{\varepsilon}_{\mathrm{s}}$ for gold and silver in a water background, $N_{\mathrm{b}}=1.33$, as the vacuum

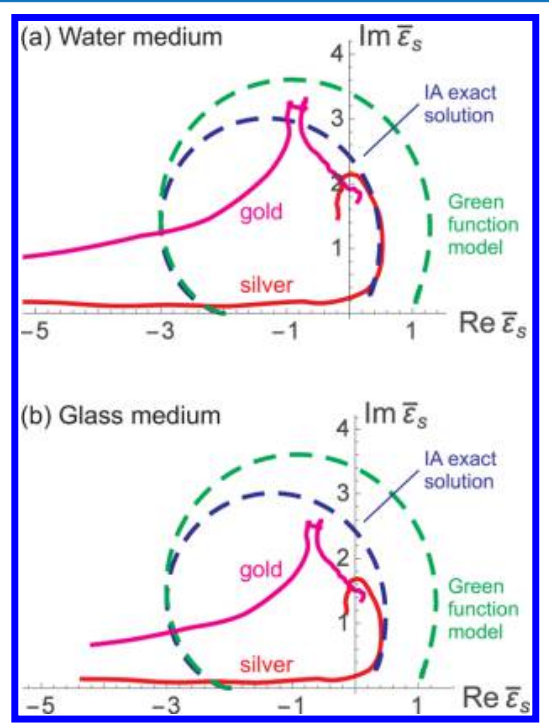

Figure 4. Evolution of the normalized permittivity $\bar{\varepsilon}_{\mathrm{s}}=\varepsilon_{\mathrm{s}} / \varepsilon_{\mathrm{b}}$ in the complex plane as the vacuum wavelength is varied for silver nanoparticles (red) $\lambda_{v} \in\{188-500\} \mathrm{nm}$ and gold nanoparticles (magenta) $\lambda_{v} \in\{188-600\} \mathrm{nm}$ embedded in a water medium $N_{\mathrm{b}}=$ 1.33 (a), and a glass medium $N_{\mathrm{b}}=1.5$ (b). The values of $\bar{\varepsilon}_{\mathrm{s}}$ required to reach ideal absorption in the lowest electric dipole mode are plotted in dashed blue for $k R$ varying from 0 to 4 . The intersections between this curve and the measured metal permittivity provide the conditions to optimize absorption using silver or gold nanoparticles. Tables 1 and 2 detail these conditions for water and glass media. For comparison, we also represent the Green function point-like predictions of eq 12 in dashed green. As already noted in Figure 1, this model fails in accurately predicting the IA conditions.

wavelength of light varies from the near-ultraviolet to the midvisible range (using interpolated Johnson and Christy data $\left.{ }^{36}\right)$. The values of $\bar{\varepsilon}_{\text {s }}$ required for producing the lowest electric dipole IA are also plotted in this figure as the size parameter, $k R$, varies from 0 to 4 . The values predicted for IA by the Green function point-like model are also plotted in this graph as $k R$ varies from 0 to infinity. IA is predicted to occur at the values of $k R$ and frequencies where the IA curves, dashed lines, intercept the experimentally determined dispersion relations, full lines.

Inspection of Figure $4 \mathrm{a}$ indicates three possible IA solutions in a water background (cf. Table 1), we caution that only the
Table 1. Conditions to Reach Electric Dipole IA for Silver and Gold Nanoparticles in Water Medium $\left(N_{b}=1.33\right)$ : Particle Diameters $D_{\text {IA }}$, Vacuum Wavelengths $\lambda_{v}^{(\mathrm{IA})}$, and NonNormalized Metal Permittivity $\boldsymbol{\varepsilon}_{\mathrm{s}}$

\begin{tabular}{cccc}
$D_{\mathrm{IA}}(\mathrm{nm})$ & $\lambda_{v}^{(\mathrm{IA})}(\mathrm{nm})$ & $k R_{\mathrm{IA}}$ & \multicolumn{1}{c}{$\varepsilon_{\mathrm{Ag}}$} \\
34.04 & 393.76 & 0.361 & $-4.094+i 0.199$ \\
115.53 & 295.80 & 1.63 & $0.704+i 3.01$ \\
291.4 & 321.0 & 3.13 & $0.700+i 0.856$ \\
$D_{\mathrm{IA}}(\mathrm{nm})$ & $\lambda_{v}^{(\mathrm{IA})}(\mathrm{nm})$ & $k R_{\mathrm{IA}}$ & $\varepsilon_{\mathrm{Au}}$ \\
91.74 & 540.69 & 0.709 & $-5.30+i 2.20$ \\
121.59 & 451.81 & 1.12 & $-1.76+i 5.27$ \\
75.94 & 273.38 & 1.16 & $-1.37+i 5.17$
\end{tabular}

lowest particle sizes correspond to resonances dominated by a dipolar response. Dipole IA solutions at larger particles sizes will include significant absorption in higher order modes similar to that seen in Figure 3c,d.

Analogous curves are plotted in Figure $4 \mathrm{~b}$ for a higher background index material, $N_{\mathrm{b}}=1.5$, like that typical of polymers and glass. At this higher index background medium, only one IA solution exists for both silver and gold. The IA values for gold and silver deduced from an analysis of the intercepts between the IA conditions and the experimental permittivity functions of Figure 4 are given in Tables 1 and 2 for $N_{\mathrm{b}}=1.33$ and $N_{\mathrm{b}}=1.5$, respectively.

Table 2. Conditions to Reach Electric Dipole IA for Silver and Gold Nanoparticles in Glass Medium $\left(N_{b}=1.5\right)$ : Particle Diameters $D_{\text {IA }}$, Vacuum Wavelengths $\lambda_{v}^{(\mathrm{IA})}$, and NonNormalized Metal Permittivity $\varepsilon_{\text {s }}$

\begin{tabular}{cccc}
$D_{\mathrm{IA}}(\mathrm{nm})$ & $\lambda_{v}^{(\mathrm{IA})}(\mathrm{nm})$ & $k R_{\mathrm{IA}}$ & $\varepsilon_{\mathrm{Ag}}$ \\
30.62 & 412.77 & 0.35 & $-5.16+i 0.227$ \\
$D_{\mathrm{IA}}(\mathrm{nm})$ & $\lambda_{v}^{(\mathrm{IA})}(\mathrm{nm})$ & $k R_{\mathrm{IA}}$ & $\varepsilon_{\mathrm{Au}}$ \\
76.23 & 559.68 & 0.64 & $-6.57+i 1.95$ \\
\hline
\end{tabular}

Although IA is predicted for both silver and gold in transparent material media, one can expect significant differences in their optical response in view of the considerable differences in their respective dispersion relations and predicted IA sizes. This is indeed the case, as illustrated in Figure 5 where the scattering and absorption cross sections of the silver (a) and (b) and gold particles (c) and (d) are plotted for frequencies in the visible range for particles whose IA diameters are taken from Table $2\left(D_{\text {IA }}=30.6 \mathrm{~nm}\left(\lambda_{v}=413 \mathrm{~nm}\right)\right.$ for silver, and $D_{\text {IA }}$ $=76.2 \mathrm{~nm}\left(\lambda_{v}=560 \mathrm{~nm}\right.$ for gold $\left.)\right)$.

The frequencies at which IA is predicted are indicated by vertical dashed lines, and the IA cross sections of $8 \pi \sigma_{\mathrm{abs}} /\left(3 \lambda^{2}\right)$ $=1$ are indicated by horizontal dashed lines in these figures. The total absorption and scattering cross sections are drawn in blue, and electric dipole contributions are drawn in red, but these curves are nearly indistinguishable on the scale of these graphs except for short wavelengths in gold. Magnetic dipole contributions are plotted in green, but are negligible in all graphs excepting some mild magnetic dipole absorption in gold at short wavelengths.

In Figure 5, one remarks significant differences between the respective behaviors of silver and gold IA particles near their IA resonances. Notably, the electric quadrupole, magnetic dipole and higher order corrections are essentially negligible near the silver IA resonance, while nonelectric dipole orders contribute significantly to absorption (but not scattering) at wavelengths 


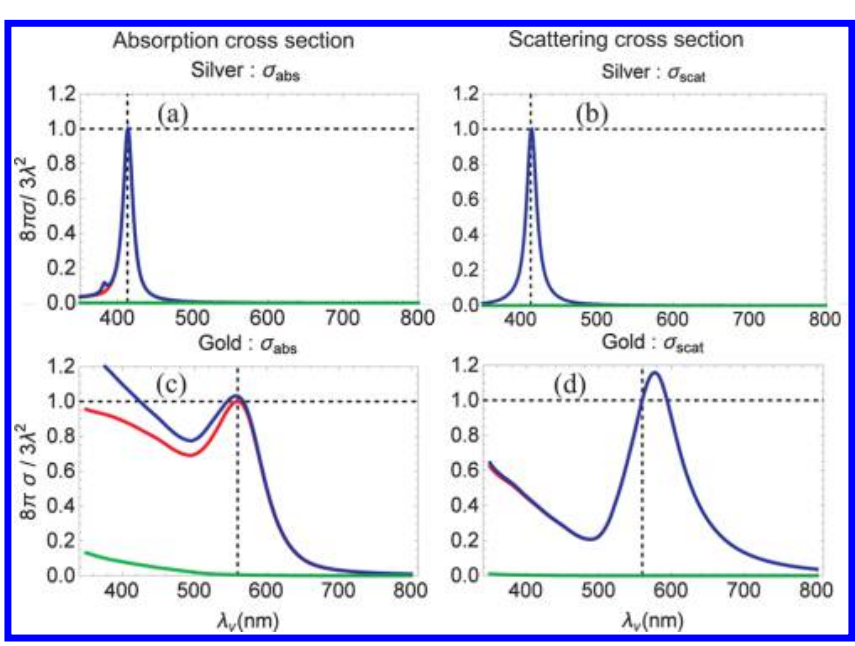

Figure 5. Absorption (a) and scattering (b) cross sections for silver nanoparticles designed to reach IA in glass medium $N_{\mathrm{b}}=1.5$ (blue curves). Dipole contributions to the cross sections are plotted in red for the electric dipole and green for the magnetic dipole. The silver nanoparticle diameter is $D_{\mathrm{IA}}=30.6 \mathrm{~nm}$. The IA wavelength is specified by a vertical dashed line, and corresponds to the situation of the cross section reaching the limit of eq 6. (c, d) Same as (a, b) for gold nanoparticles of diameter $D_{\mathrm{IA}}=76.2 \mathrm{~nm}$.

below the IA resonance in gold. One should also remark that, although the dipole contributions to the absorption cross sections are limited by their theoretical upper bound of $\sigma_{\mathrm{abs}}=$ $3 \lambda^{2} /(8 \pi)$, the electric dipole scattering cross section of gold rises to values above $3 \lambda^{2} /(8 \pi)$, at frequencies below the IA resonance, but this is allowed by the general theoretical considerations of 1 where one sees that the scattering cross section of a dipole mode is only required by unitarity to satisfy $\sigma_{\text {scat }} \leq 3 \lambda^{2} /(2 \pi)$. Nevertheless, one can see in both Figure $5 \mathrm{~b}$ and Figure $5 \mathrm{~d}$ that $\sigma_{\text {scat }}=3 \lambda^{2} /(8 \pi)$ at the IA resonance as required by the general theoretical restraint of eq 6 .

\section{IDEAL ABSORPTION FOR CORE-SHELL NANOPARTICLES}

Although we saw that changing the background material index allows some control over IA frequency with homogeneous inclusions, applications are likely to request IA to occur at particular frequencies with a set of available materials. This requires additional adjustable geometric parameters and concentric coatings is one of the simplest ways to achieve this.

If we consider particles consisting of a spherical core and a single concentric coating, one has two adjustable parameters, the radius of the outer shell $R$, and its concentric spherical core, $R_{c}$ (cf. Figure 6). It proves convenient to fix these two

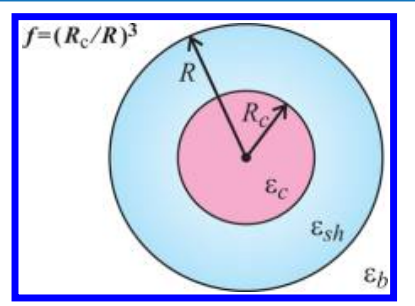

Figure 6. Schematic of a concentric coated sphere where the core, shell, and background permittivities are denoted, respectively, $\varepsilon_{\mathcal{c}}, \varepsilon_{\mathrm{sh}}$, and $\varepsilon_{\mathrm{b}}$. The filling fraction of the core material is defined as $f \equiv\left(R_{\mathrm{c}}\right)$ $R)^{3}$. dimensioned parameters, $R$ and $R_{c}$, in terms of the dimensionless size parameter, $k R$, and core material filling factor $f \equiv\left(R_{\mathrm{c}} / R\right)^{3}$. Unlike the homogeneous sphere studied in the previous section, the analytic properties of the $S$ matrix cannot be directly exploited to determine the parameters producing IA solutions, since no IA solutions are guaranteed to exist when varying the parameters $k R$ and $f$ of a coated sphere with fixed permittivities.

Given the complexity of the analytic coated sphere solutions, the possible nonexistence of solutions at finite frequency, and finally the fact that there are multiple adjustable parameters, it is advantageous to develop compact approximate formulas that allow one to rapidly determine which parameters and materials can provide viable designs for coated sphere ideal absorbers. We derive now an approximation which allows the design of core-shell electric dipole IA scatterers by taking advantage of the fact that one is generally interested in designing subwavelength IA particles.

We saw that eq 16a provides an accurate approximation for the required IA permittivity contrast, $\bar{\varepsilon}_{s}$, over the entire range of the size parameter $\rho=k R$. The Maxwell-Garnett effective medium approach is derived as the effective permittivity of a concentric subwavelength core-shell particle whose volume fraction matches that of the bulk material. The effective permittivity, $\bar{\varepsilon}_{\text {eff, }}$ of a core-shell system can thus be written as a function of filling fraction, $f:{ }^{37}$

$$
\bar{\varepsilon}_{\mathrm{eff}}=\bar{\varepsilon}_{\mathrm{sh}}\left(1+3 f \frac{\bar{\varepsilon}_{\mathrm{c}}-\bar{\varepsilon}_{\mathrm{sh}}}{\bar{\varepsilon}_{\mathrm{c}}+2 \bar{\varepsilon}_{\mathrm{sh}}-f\left(\bar{\varepsilon}_{\mathrm{c}}-\bar{\varepsilon}_{\mathrm{sh}}\right)}\right)
$$

where $\bar{\varepsilon}_{\mathrm{sh}}$ and $\bar{\varepsilon}_{\mathrm{c}}$ are the shell and core relative permittivities, respectively. This equation can then be algebraicallv inverted to yield filling fraction as a function of $\bar{\varepsilon}_{\text {eff }}$ that is: $\Omega$

$$
f=\frac{\varepsilon_{c}+2 \varepsilon_{\mathrm{sh}}}{\varepsilon_{c}-\varepsilon_{\mathrm{sh}}} \frac{\varepsilon_{\mathrm{eff}}-\varepsilon_{\mathrm{sh}}}{\varepsilon_{\mathrm{eff}}+2 \varepsilon_{\mathrm{sh}}}
$$

One can then solve for core-shell IA by replacing the "effective" index in eq 18 by the $\bar{\varepsilon}^{(e)}$ of eq 16a that specifies the permittivity required for the IA condition. Since $\bar{\varepsilon}_{\text {eff }}$ and at least one of the materials is complex valued, the filling fraction, $f$, found by eq 18 is generally complex, but one can vary the size parameter, $\rho=k R$ to obtain a $\mathcal{I}\{f\}=0$ solution graphically. Provided that the real part of the filling fraction is less than 1 when $\mathcal{I}\{f\}=0$, then one has found a valid IA solution.

We apply the graphic solution method described in the previous paragraph to the core-shell IA design consisting of a silver or gold metallic layer around a lossless high index core of $N_{\mathrm{c}}=2.8$ in a background medium of index $N_{\mathrm{b}}=1.5$. The results are illustrated in Figure 7. The filling fractions of the shell material and the radius of the particle are given as a function of the vacuum wavelength of the incident field. We remark that the chosen core-shell design with a high index center and a metallic shell allows one to design IA for any wavelength higher than the homogeneous sphere solutions given in Table 2. It is also possible to design IA for other geometries, like a dielectric shell around a metallic core, but the metallic shell design illustrated here tended to give the smallest IA solutions and seemed more realizable from a practical standpoint.

\section{CONCLUSIONS}

In this work, we have derived simple analytical expressions, eqs (16), to accurately predict the lowest order electric and magnetic modes leading to Ideal Absorption (IA). These 


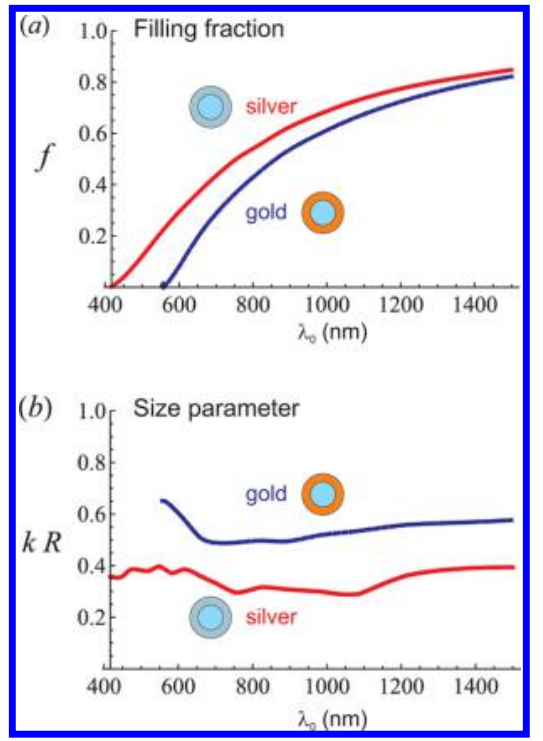

Figure 7. Plots of the filling fraction (a) and the size parameter (b), required to achieve IA in a core-shell structure immersed in a $N_{b}=$ 1.5 background material. The core is considered to be a lossless high index material, $N_{c}=2.8$, and the shell is either silver (red line) or gold (blue line).

expressions provide a significant improvement as compared to the classical "point-like" models derived using Green's function or Mie coefficients expansion, and can accurately handle nanoparticle sizes up to several wavelengths. The IA criteria was shown to correspond to a limit behavior of an absorption cross section channel. Precise electromagnetic calculations in the complex frequency plane were also introduced to predict this phenomenon, indicating the intriguing possibility of observing IA in high index absorbing materials and not just in metals. Our results showed that IA should be observable at visible frequencies for homogeneous nanoparticles composed of gold or silver for specific (fixed) frequencies and sizes. The methods and formulas developed in this work led to a simple scheme (eq 18) for predicting core-shell geometries supporting effective IA conditions throughout the visible spectrum. Experimental observations of IA for optimized subwavelength particles are within reach and should prove useful to better understand IA and its signature in quantum systems.

\section{AUTHOR INFORMATION}

\section{Corresponding Author}

*E-mail: brian.stout@fresnel.fr.

\section{Notes}

The authors declare no competing financial interest.

\section{ACKNOWLEDGMENTS}

The authors would like to thank Guillaume Baffou and Xavier Zambrana for interesting discussions and helpful remarks. The research leading to these results has received funding from the European Research Council under the European Union's Seventh Framework Programme (FP7/2007-2013)/ERC Grant Agreements 278242 (ExtendFRET), and from the A*MIDEX Project (No. ANR-11-IDEX-0001-02) funded by the Investissements d'Avenir French Government program managed by the French National Research Agency (ANR).

\section{REFERENCES}

(1) Lal, S.; Link, S.; Halas, N. Nano-optics from sensing to waveguiding. J. Nat. Photonics 2007, 2007 (1), 64-648.

(2) Schuller, J. A.; Barnard, E. S.; Cai, W. S.; Jun, Y. C.; White, J. S.; Brongersma, M. L. Plasmonics for extreme light concentration and manipulation. Nat. Mater. 2010, 9, 193-204.

(3) Novotny, L.; van Hulst, N. Antennas for light. Nat. Photonics 2011, 5, 83-90.

(4) Atwater, H. A.; Polman, A. Plasmonics for improved photovoltaic devices. Nat. Mater. 2010, 9, 205-213.

(5) Baffou, G.; Quidant, R. Thermo-plasmonics: using metallic nanostructures as nano-sources of heat. Laser Photonics Rev. 2013, 7, 171-187.

(6) Mayer, K. M.; Hafer, J. H. Localized surface plasmon resonance sensors. Chem. Rev. 2011, 111, 3828-3857.

(7) Halas, N. J.; Lal, S.; Chang, W. S.; Link, S.; Nordlander, P. Plasmons in strongly coupled metallic nanostructures. Chem. Rev. 2011, 111, 3913-3961.

(8) Baffou, G.; Quidant, R. Nanoplasmonics for chemistry. Chem. Soc. Rev. 2014, 43, 3898.

(9) Chong, Y. D.; Ge, Li; Cao, H.; Stone, A. D. Coherent perfect absorbers: time-reversed lasers. Phys. Rev. Lett. 2010, 105, 053901.

(10) Noh, H.; Chong, Y.; Stone, A. D.; Cao, H. Perfect coupling of light to surface plasmons by coherent absorption. Phys. Rev. Lett. 2012, 108, 186805.

(11) Noh, H.; Popoff, S. M.; Cao, H. Broadband subwavelength focusing of light using a passive sink. Opt. Express 2013, 21, 1743517446 .

(12) Sentenac, A.; Chaumet, P. C.; Leuchs, G. Total absorption of light by a nanoparticle: an electromagnetic sink in the optical regime. Opt. Lett. 2013, 38, 818-820.

(13) Grigoriev, V.; Tahri, A.; Varault, S.; Rolly, B.; Stout, B.; Wenger, J.; Bonod, N. Optimization of resonant effects in nanostructures via Weierstrass factorization. Phys. Rev. A 2013, 88, 011803.

(14) Grigoriev, V.; Varault, S.; Boudarham, G.; Stout, B.; Wenger, J.; Bonod, N. Singular analysis of Fano resonances in plasmonic nanostructures. Phys. Rev. A 2013, 88, 063805.

(15) Tsang, L.; Kong, J. A.; Ding, K.-H. Scattering of Electromagnetic Waves; Wiley Series in Remote Sensing; John Wiley \& Sons: New York, 2000; Vol.1.

(16) Stout, B.; Auger, J. C.; Devilez, A. Recursive T matrix algorithm for resonant multiple scattering: Applications to localized plasmon excitations. J. Opt. Soc. Am. A 2008, 25, 2549-2557.

(17) Newton, R. G. Scattering Theory of Waves and Particles; McGraw-Hill: New York, 1966.

(18) van de Hulst, H. C. Light Scattering by Small Particles; Dover Publications: Mineola, NY, 1957.

(19) Bohren, C. F.; Huffman, D. R. Absorption and Scattering of Light by Small Particles; Wiley-Interscience: New York, 1983.

(20) Fleury, R.; Soric, J.; Alù, A. Physical bounds on absorption and scattering for cloaked sensors. Phys. Rev. B 2014, 89, 045122(12).

(21) Tretyakov, S. Maximizing absorption and scattering by dipole particles. Plasmonics 2014, 9, 935-944.

(22) Nieminen, T. A.; Rubinsztein-Dunlop, H.; Heckenberg, N. R. Calculation of the T-matrix: General considerations and application of the point-matching method. J. Quant. Spectrosc. Radiat. Transfer 2003, 79-80, 1019-1029.

(23) Botten, L. C.; McPhedran, R. C.; Nicorovici, N. A.; Derrick, G. H. Periodic models for thin optimal absorbers of electromagnetic radiation. Phys. Rev. B 1997, 55, R16072-R16075.

(24) Sarychev, A. K.; Bergman, D. J.; Yagil, Y. Theory of the optical and microwave properties of metal-dielectric films. Phys. Rev. B 1995, $51,5366-5385$.

(25) de Vries, P.; van Coevorden, D. V.; Lagendijk, A. Point scatterers for classical waves. Rev. Mod. Phys. 1998, 70, 447-466.

(26) Pellegrini, Y.-P.; Thibaudeau, P.; Stout, B. Off-shell mean-field electromagnetic T-matrix of finite size spheres and fuzzy scatterers. J. Phys.: Condens. Matter 1997, 9, 177-191. 
(27) Moroz, A. Depolarization field of spheroidal particles. J. Opt. Soc. Am. B 2009, 26, 517-527.

(28) Le Ru, E. C.; Somerville, W. R. C.; Auguié, B. Radiative correction in approximate treatments of electromagnetic scattering by point and body scatterers. Phys. Rev. A 2013, 87, 012504.

(29) Albaladejo, S.; Gómez-Medina, R.; Froufe-Pérez, L. S.; Marinchio, H.; Carminati, R.; Torrado, J. F.; Armelles, G.; GarciaMartin, A.; Sáenz, J. J. Radiative corrections to the polarizability tensor of an electrically small anisotropic dielectric particle. Opt. Exp. 2010, 70, 3563.

(30) Colas des Francs, G.; Girard, C.; Bruyant, A.; Dereux, A. SNOM signal near plasmonic nanostructures: An analogy with fluorescence decay channels. J. Microsc. 2007, 229, 302-306.

(31) Colas des Francs, G.; Bouhelier, A.; Finot, E.; Weeber, J. C.; Dereux, A.; Girard, C.; Dujardin, E. Fluorescence relaxation in the near-field of a mesoscopic metallic particle: distance dependence and role of plasmon modes. Opt. Express 2008, 16, 17654-17666.

(32) Stratton, J. A. Electromagnetic Theory; Wiley: New York, 1947; IEEE Press reissue 2007.

(33) Stout, B.; Devilez, A.; Rolly, B.; Bonod, N. Multipole methods for nanoantennas design: applications to Yagi-Uda configurations. $J$. Opt. Soc. Am. B 2011, 28, 1213-1223.

(34) Watson, G. N. A Treatise on the Theory of Bessel Functions; Cambridge University Press: Cambridge, 1980.

(35) Asenjo-Garcia, A.; Manjavacas, A.; Myroshnychenko, V.; García de Abajo, F. J. Magnetic polarization in the optical absorption of metallic nanoparticles. Opt. Express 2012, 20, 28142-28152.

(36) Johnson, P. B.; Christy, R. W. Optical constants of the noble metals. Phys. Rev. B 1972, 6, 4370-4379.

(37) Choy, T. Effective Medium Theory, 1st ed.; Oxford University Press: Oxford, 1999; Vol. 20, ISBN 978-0-19-851892-1.

\section{NOTE ADDED AFTER ASAP PUBLICATION}

This paper was originally published on February 5, 2015, with an error in Equation 16a. The corrected version was reposted on February 9, 2015. 